\title{
Machine Learning for Sub-pixel Super-resolution in Direct Electron Detectors
}

\author{
Gabriela Correa and David Muller
}

Cornell University, Ithaca, New York, United States

Direct electron detectors (DED) are now able to image biological specimens with higher resolution than $\mathrm{X}$-rays - and are enabling new materials imaging techniques such as 4D-STEM. Limitations imposed by semiconductor manufacturing processes, where consumer electronics dominate device chip surface area, reduce the available space for DED pixels. To push beyond the physical pixel, sub-pixel super-resolution is necessary. We explore the prospects for sub-pixel super-resolution through electron counting as a function of diode depth, pixel pitch, and beam energy. For most energy ranges of interest to electron microscopists, energy is deposited as a string of charge across multiple pixels. We use machine learning to identify the start of the string, determining the true entry point of an electron with greater success than existing electron counting statistics.

To test the effectiveness of different electron counters across beam energies and DED architectures (Fig. 1a), we feed the electron counter virtual detector readouts. Three electron counters are tested: the maximum intensity (peak) pixel; the center of mass (mean) point; and a convolutional neural network with a rectified linear unit (ReLU). We simulated primary beam energies from 30 to 5,000 keV for silicon and germanium diodes, with pixel pitches from 1 to $500 \mu \mathrm{m}$ and diode depths from 10 to 1,000 $\mu \mathrm{m}$. Electron paths are generated through an electron Monte Carlo method [1] with relativistic corrections [2] (Fig. 1b), then projected into a range of virtual detector pixels. The root mean squared error between the true entry point and the counter's guessed entry point is used as a metric of performance. The simulations are performed, assuming a perfect signal with no additional noise, to test the maximum capability of counter performance.

Super-resolution counting is effective up to $300 \mathrm{keV}$ for a $500 \mu \mathrm{m}$ diode as in the PAD [3], and up to 100 $\mathrm{keV}$ for shallower diode depth of $50 \mu \mathrm{m}$ as in a MAPS [4]. The machine learning model has great performance with a training dataset of significantly smaller size than a typical 4D-STEM dataset (Fig 2a). Similar trends for all counters are observed with respect to beam energy, pixel pitch, and diode depth (Fig. 2b). The electron counters are generally most dependent on diode depth for performance, followed by beam energy. There are three regions of dependencies on diode depth: a barreling region for backthinned diodes where the electron passes straight through; a peak error region where the electron path is cut off but given sufficient time to wander; and a region where the full point spread function is captured. A single path projected onto pixels (Fig. 2c) is sparsified to a single-entry coordinate through all electron counters. In general, tracking the peak-energy pixel has the highest error, closely followed by center of mass. Our machine-learning-based electron counting algorithm has the highest performance. By combining machine learning with a deep diode, a counting mode via integration is achieved. [5] 
(a)

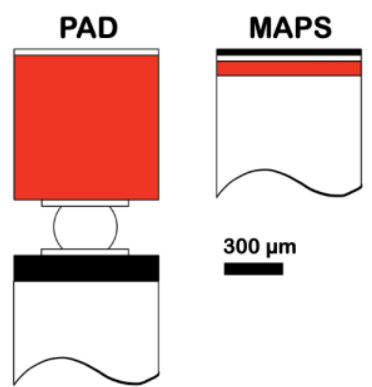

(b)

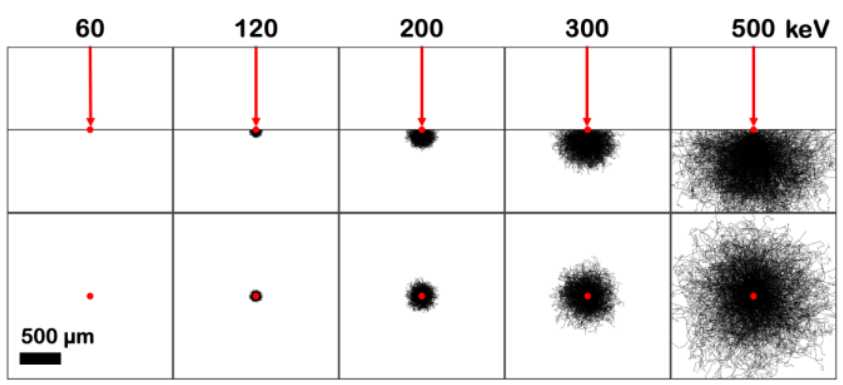

Figure 1. (a) Detector geometries for PAD (left) and MAPS (right) with distinctly different diode depths. Diodes are shaded red, while readout electronics are highlighted in black. (b) Monte Carlo simulations of electron paths (only 1000 shown) at varying beam energies. With higher energy, the electron has greater capability to travel far from where it started. The top row contains a projected side view of the electron paths incident on the surface, while the bottom row is a plan view projection. Red dots indicate electron entry points.

(a)

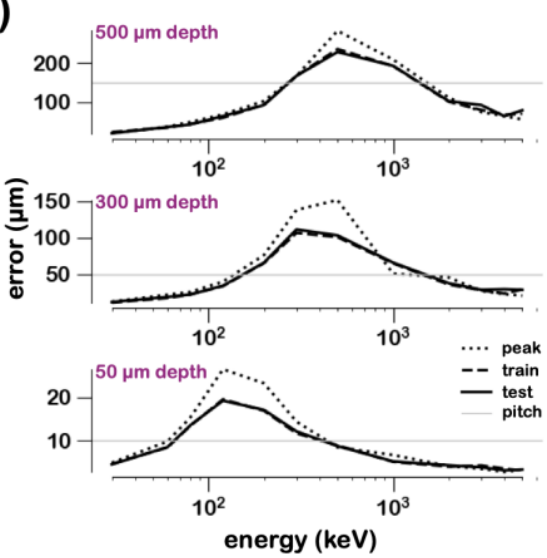

(b)
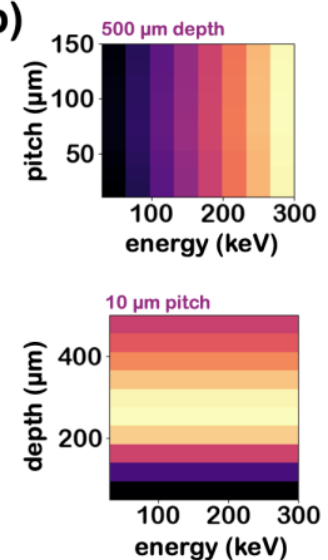
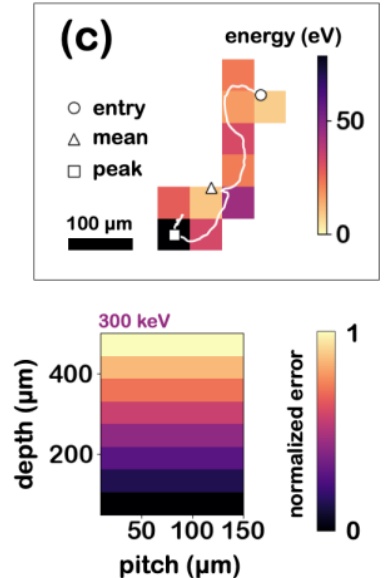

Figure 2. (a) Error with respect to energy for three different detector geometries. Pixel pitch is shown as a silver horizontal line, in each plot labeled by a diode depth. Super-resolution regimes are shown, as the regions where the error is less than the pixel pitch. The training and test performance of the machinelearning-based electron counter is shown, in comparison to the peak pixel calculated simultaneously. (b) Data cube illustrating normalized error dependencies on pixel pitch, beam energy, and diode depth; slices of the center of mass data show great dependency on diode depth followed by beam energy. (c) A single electron path with its overlaid detector readout, at $300 \mathrm{keV}$ in a $500 \mu \mathrm{m}$ deep diode with $50 \mu \mathrm{m}$ pitch. The summation of all pixels is the energy of the incident electron, for sufficient diode depth. Different counting metrics for this particular path are labeled.

\section{References}

[1] D. C. Joy, Monte Carlo modeling for electron microscopy and microanalysis, vol. 9. Oxford University Press, 1995. 
[2] T. A. Caswell, P. Ercius, M. W. Tate, A. Ercan, S. M. Gruner, and D. A. Muller, "A high-speed area detector for novel imaging techniques in a scanning transmission electron microscope," Ultramicroscopy, vol. 109, no. 4, pp. 304-311, Mar. 2009.

[3] M. W. Tate et al., "High Dynamic Range Pixel Array Detector for Scanning Transmission Electron Microscopy," Microsc. Microanal., vol. 22, no. 1, pp. 237-249, Feb. 2016.

[4] M. Battaglia et al., "A rad-hard CMOS active pixel sensor for electron microscopy," Nucl. Instruments Methods Phys. Res. Sect. A Accel. Spectrometers, Detect. Assoc. Equip., vol. 598, no. 2, pp. 642-649, 2009.

[5] This work used resources provided by the National Science Foundation Platform for the Accelerated Realization, Analysis, and Discovery of Interface Materials (PARADIM) under Cooperative Agreement No. NSF-DMR-1539918, and resources of the National Energy Research Scientific Computing Center (NERSC), a U.S. Department of Energy Office of Science User Facility operated under Contract No. DEAC02-05CH11231. GCC acknowledges support by the Alfred P. Sloan Foundation and Department of Energy Computational Science Graduate Fellowship (DOE CSGF), which is provided under grant number DE-FG02-97ER25308. 